\title{
Clinical and molecular analyses of thyroid cancer in patients treated for benign diseases
}

\section{Dear Editor}

Incidental thyroid carcinoma (ITC) is defined as a tumor diagnosed at histology after surgical intervention for a benign thyroid disease and, therefore, without a preoperative diagnosis of malignancy. The prevalence of ITCs reported in the 20 papers of the literature is extremely variable (3.3-21.6\%), mostly due to the relatively low number of cases included (Ugolini et al. 2007, Bradly et al. 2009, Barczyński et al. 2011, Minuto et al. 2013, Negro et al. 2013), and scanty molecular data are available (Ugolini et al. 2007). A more accurate estimation of the actual prevalence of ITCs is of particular importance for the preoperative counseling of patients undergoing thyroidectomy for a supposed benign disease. Moreover, the definition of the clinical behavior of this category of tumors is crucial for the choice of the best postsurgical treatments and follow-up.

The aim of this study was to investigate the prevalence, clinical features, and BRAF mutational status of ITCs of any dimension in a large consecutive cohort of patients referred to our Endocrine Surgery Center for benign thyroid disease. The approval of the Ethical Committee was obtained for this retrospective observational study (\#139/2013).

Between February 1997 and March 2011, 2045 patients were subjected to thyroidectomy at the Endocrine Surgery Unit for benign disease. The preoperative diagnosis was uninodular or multinodular goiters (MNGs) in 1405 cases, toxic uninodular goiter or toxic MNGs (TMNGs) in 332 cases, Graves' disease (GD) in 300 cases, Hashimoto's thyroiditis in six cases, and iatrogenic thyrotoxicosis in two cases. The preoperative diagnosis was based on a benign cytology observed during ultrasound-guided fineneedle aspiration biopsy (FNAB) performed on either thyroid nodules with suspicious ultrasound features or the largest thyroid nodule if no suspicious features were detected. In TMNGs, only nodules with a normal or reduced uptake were subjected to FNAB. All cases with an indeterminate cytology were excluded. The surgical treatment was total thyroidectomy in $96.5 \%$ of the cases. In 42 cases, suspicious lymph nodes were observed during intervention and lymphadenectomy of the central neck compartment (VI level) was performed. Tumors were classified according to the thyroid malignancy World Health Organization classification and staged according to the sixth edition of the TNM staging. Only tumors with an extrathyroidal invasion (i.e. invasion of the thyroid capsule and/or lymph nodes or distant metastases) or with a diameter $>2 \mathrm{~cm}$ were subjected to radioiodine residue ablation. Criteria used to identify remission or persistent/ recurrent disease were drawn on the basis of International guidelines for the management of differentiated thyroid cancer (American Thyroid Association (ATA) et al. 2009).

Neoplastic cells were obtained by laser microdissection from the tumor tissue of patients with a diagnosis of incidental papillary thyroid cancers (PTCs) and $B R A F^{V 600 E}$ mutation was analyzed by PCR amplification and direct sequencing, as described previously (Fugazzola et al. 2006).

Several outcome predictors were evaluated by univariate and multivariate analyses, by means of $\chi^{2}$-test, $t$-test, or stepwise multiple regression, as appropriate. Statistical significance was defined as $P<0.05$. All statistical analyses were performed using SPSS 8.0 statistical package for Windows (SPSS, Inc.).

Among the 2045 consecutive cases subjected to thyroidectomy without a preoperative diagnosis of malignancy, ITCs were found in 249 patients (12.2\%). In particular, the prevalence was $13.4,9.9$, and $9.3 \%$ in MNGs, TMNGs, and GD respectively (Fig. 1). Moreover, ITCs were classified according to the year of surgery, and a progressive increase in their prevalence, from $8.4 \%$ in 1998 to $16.5 \%$ in 2010, was found. The overall mean prevalence obtained considering the 20 studies reported in the literature was $9.3 \%$, being $9 \%$ in MNGs, $7.2 \%$ in TMNGs, and $6.7 \%$ in GD.

The clinical and histopathological features of the 249 ITCs are reported in Table 1. In particular, the mean age

Published by Bioscientifica Ltd. 


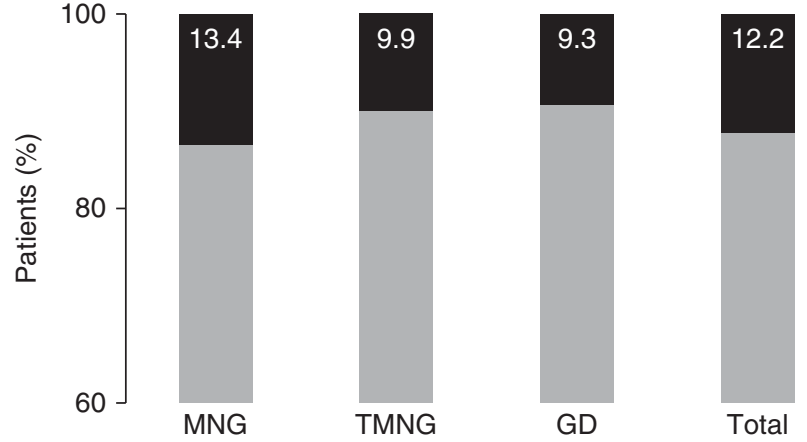

Figure 1

Prevalence of incidental thyroid cancers (ITCS) in the different categories. The black part of the column indicates the percentage of ITCs. MNG, normo-functioning nodular goiter; TMNG, toxic nodular goiter; GD, Graves' disease.

was 51.7 years (median 52 years) and the F:M ratio was 4:1. The mean tumor diameter was $9.5 \mathrm{~mm}$ (median $6 \mathrm{~mm}$ ), and the papillary histotype was the most prevalent $(91.6 \%)$. Most cases were classified as T1 (81.9\%) and stage I (86.7\%); the percentage of infiltrating tumors was around $12 \%$; and multicentricity was found in $36 \%$ of the cases. In 16 of the 249 tumor cases $(6.4 \%)$, the diameter of the malignant nodule was $\geq 30 \mathrm{~mm}$. In these cases, the preoperative diagnoses were MNGs in 14 cases and TMNGs in 2 cases, and the histological diagnoses were follicular cancer in ten cases, medullary cancer in one case, and papillary cancer in the remaining five cases. This high prevalence of follicular malignant lesions among the larger nodules of this series could account for the preoperative diagnosis of benignity found in the nodules $(12 / 16)$ subjected to FNAB. Concerning the TMNG category, in 24 of the 33 cases found to have thyroid cancer at histology, FNAB was not performed since the hyperfunctioning nodule was only visible during ultrasound $(n=3)$ or because the diameter of the nodules with a normal or reduced uptake was $<7 \mathrm{~mm}$ without suspicious features during ultrasound.

The 175 of 249 patients for whom follow-up data were available were divided according to the remission/persistence of the disease after a mean follow-up of more than 8 years (median 107 months). The large majority of patients $(89.7 \%)$ were in remission at the last follow-up visit. The clinical features found to be significantly associated with the persistence of the disease were the tumor diameter $(P=0.03)$, tumor extension $(P=0.0004)$, and stage $(P=0.001)$. During the stepwise multiple regression analysis, only stage was confirmed to be statistically significant $(P<0.001$, data not shown).

The molecular analysis of $B R A F$ was done in 134 of 228 incidental PTC cases, and it revealed a mutation in 12 cases (9\%). Among the several clinical parameters, such as age, gender, tumor extension, multifocality, and stage, $B R A F^{V 600 E}$ mutation was found to be associated only with a larger tumor diameter $(P=0.02$, data not shown). Accordingly, on classification of pT1 tumors, BRAF was less frequently mutated in pT1a tumors with respect to pT1b tumors ( 6 vs $18 \%, P=0.07$ ). Similarly, on separating tumors with a diameter $\leq 10 \mathrm{~mm}$ from the larger tumors of any diameter, the prevalence of $B R A F^{V 600 E}$ mutation was found to be significantly higher in the latter group (6 vs $21 \%, P=0.02$ ).

Table 1 Clinical and histopathological features of the 249 incidental thyroid cancer cases. Owing to the absence of a preoperative diagnosis of malignancy, lymphadenectomy was performed only in a minority of cases $(n=42)$, when suspicious lymph nodes were observed intraoperatively.

\section{Features}

Age (years)

Mean

Median

Range

Tumor diameter $(\mathrm{mm})$

Mean

Median

Range

Gender (female)

51.7

52

$17-83$

Tumor

T1

$\mathrm{T} 2$

T3

T4

Multicentricity

Lymph nodes

NX

NO

N1

Stage

I

II

III

IV

Histotype

Papillary

Follicular

Medullary

$\mathrm{NH}$ lymphoma

9.5

6

1-70

$n(\%)$

195 (78.3)

204 (81.9)

15 (6)

24 (9.6)

6 (2.4)

89 (35.9)

206 (83.1)

$26(10.5)$

16 (6.4)

216 (86.7)

5 (2)

$22(8.8)$

6 (2.4)

228 (91.6)

$18(7.2)$

$2(0.8)$

$1(0.4)$

Histological variant papillary carcinoma

Classic

$194(82.9)$

Follicular

Oncocytic

$33(14.1)$

$3(1.3)$

$4(1.7)$

Histological variant follicular carcinoma

Classic

$12(66.7)$

$5(27.8)$

$1(5.5)$

Insular 
In conclusion, a $12.2 \%$ overall prevalence of ITCs was found in the present large series of 2045 consecutive patients subjected to thyroidectomy for benign thyroid diseases. A lower mean prevalence $(9.3 \%)$ was found among the studies published to date on ITCs in benign thyroid diseases (Ugolini et al. 2007, Bradly et al. 2009, Barczyński et al. 2011, Minuto et al. 2013, Negro et al. 2013). The extreme variability observed among the different series could be due to the relatively low number of cases enrolled $(<1000$ in 17 of 20 series). In 71 of the 249 cases (28.5\%), tumor diameter was $>10 \mathrm{~mm}$, but the tumor was not preoperatively diagnosed due to pitfalls in the cytology or more frequently due to the presence of larger nodules in the same goiter. Thus, this finding supports the reported indication to perform FNAB in up to four nodules to exclude cancer in a MNG (Frates et al. 2006).

Interestingly, a high prevalence of ITCs was found in MNGs (13.4\%) with respect to hyperfunctioning benign diseases (9.9\% in TMNGs and $9.3 \%$ in GD), consistent with the mean data obtained from the revision of the literature (9\% in MNGs, $7.2 \%$ in TMNGs, and 6.7\% in GD). These data further confirm the results, indicating that lower levels of TSH are associated with a lower risk of PTC in patients with nodular thyroid disease (Fiore et al. 2009).

ITCs were comparable to clinically evident tumors for several features, such as age, gender, histotype, and prevalence of multifocality. The significant association between persistent disease and tumor extension and stage was also consistent with data reported for clinically diagnosed thyroid cancers. In contrast, the mean tumor diameter of ITCs was nearly $1 \mathrm{~cm}$, and a larger volume was significantly associated with a persistent disease. In the present series of ITCs, the analysis of BRAF was performed for the first time. Interestingly, though clinically similar, ITCs resulted to be different from non-incidental tumors from a molecular point of view. Indeed, the mean prevalence of $B R A F^{V 60 O E}$ mutation in non-incidental tumors was 40-50\% (Fugazzola et al. 2006, Xing 2007), whereas in the present series, the mutation was found in 12 of 134 (9\%) cases. The analysis of BRAF in ITCs has been reported previously in only one study, which was limited to 85 tumors of diameter $<4 \mathrm{~mm}$ and documented mutations in 15 of 85 (17.6\%) cases (Ugolini et al. 2007). This prevalence is similar to that observed in the present study only for TMNGs (15.4\%), with the difference in the overall frequency being probably due to the different size of the series. The lower prevalence of mutations in ITCs with respect to clinically evident cancers could be related to the lower volume of incidental cases, consistent with the present finding that $B R A F^{V 6 O O E}$ mutation is significantly associated with tumor diameter. Nevertheless, it must be considered that around $80 \%$ of all thyroid cancers measure $20 \mathrm{~mm}$ or less and that BRAF was found to be mutated in $30-40 \%$ papillary microcarcinoma cases (Xing 2007) and in $44.6 \%$ of cases in a large cohort of PTCs of diameter $<20 \mathrm{~mm}$ (Basolo et al. 2010). Thus, it is tempting to speculate that the low prevalence of $B R A F^{V 600 E}$ mutation in ITCs indicates that the majority of them harbor a low aggressiveness.

In conclusion, the analyses on the present large series indicated that ITCs have a median diameter of $6 \mathrm{~mm}$, are mostly $\mathrm{T} 1$ and stage I papillary tumors, and multifocal in around $35 \%$ of the cases, with a remission rate of $90 \%$. The low prevalence of $B R A F$ mutations further supports their indolent behavior and their reduced tendency to evolve into manifest tumors, indicating that no aggressive treatments are warranted for the majority of them.

$$
\begin{array}{r}
\text { Simone De Leo } \\
\text { Michela Perrino }^{1} \\
\text { Sara Badiali }^{3} \\
\text { Stefania Rossi }^{4} \\
\text { Valentina Cirello }^{1} \\
\text { Carla Colombo }^{1} \\
\text { Delfina Tosi } \\
\text { Dianmaria Cantoni }^{3} \\
\text { Luca Poggi }^{3} \\
\text { Gaetano Bulfamante }^{4,5} \\
\text { Paolo Beck-Peccoz }^{1,2} \\
\text { Leonardo Vicentini }^{3} \\
\text { Laura Fugazzola }^{1,2}
\end{array}
$$

${ }^{1}$ Department of Clinical Sciences and Community Health, University of Milan, Milan, Italy

${ }^{2}$ Endocrine Unit Fondazione IRCCS $\mathrm{Ca}^{\prime}$ Granda,

Via Francesco Sforza 35, 20122 Milan, Italy

${ }^{3}$ Endocrine Surgery Unit, Fondazione IRCCS $\mathrm{Ca}^{\prime}$ Granda, Via Francesco Sforza 35, 20122 Milan, Italy

${ }^{4}$ Unit of Pathological Anatomy, San Paolo Hospital Medical School, Milan, Italy

${ }^{5}$ Department of Health Science, University of Milan, Milan, Italy

(Correspondence should be addressed to L Fugazzola); email: laura.fugazzola@unimi.it

Published by Bioscientifica Ltd. 


\section{Declaration of interest}

The authors declare that there is no conflict of interest that could be perceived as prejudicing the impartiality of the research reported.

\section{Funding}

This research did not receive any specific grant from any funding agency in the public, commercial or not-for-profit sector.

\section{References}

American Thyroid Association (ATA) Guidelines Taskforce on Thyroid Nodules and Differentiated Thyroid Cancer, Cooper DS, Doherty GM, Haugen BR, Kloos RT, Lee SL, Mandel SJ, Mazzaferri EL, McIver B, Pacini F et al. 2009 Revised American Thyroid Association management guidelines for patients with thyroid nodules and differentiated thyroid cancer. Thyroid 19 1167-1214. (doi:10.1089/thy.2009.0110)

Barczyński M, Konturek A, Stopa M, Cichoń S, Richter P \& Nowak W 2011 Total thyroidectomy for benign thyroid disease: is it really worthwhile? Annals of Surgery 254 724-729. (doi:10.1097/SLA.0b013e3182360118)

Basolo F, Torregrossa L, Giannini R, Miccoli M, Lupi C, Sensi E, Berti P, Elisei R, Vitti P, Baggiani A et al. 2010 Correlation between the BRAF V600E mutation and tumor invasiveness in papillary thyroid carcinomas smaller than 20 millimeters: analysis of 1060 cases. Journal of Clinical Endocrinology and Metabolism 95 4197-4205. (doi:10.1210/jc. 2010-0337)

Bradly DP, Reddy V, Prinz RA \& Gattuso P 2009 Incidental papillary carcinoma in patients treated surgically for benign thyroid diseases. Surgery 146 1099-1104. (doi:10.1016/j.surg.2009.09.025)
Fiore E, Rago T, Provenzale MA, Scutari M, Ugolini C, Basolo F, Di Coscio G, Berti P, Grasso L, Elisei R et al. 2009 Lower levels of TSH are associated with a lower risk of papillary thyroid cancer in patients with thyroid nodular disease: thyroid autonomy may play a protective role. Endocrine-Related Cancer 16 1251-1260. (doi:10.1677/ERC-09-0036)

Frates MC, Benson CB, Doubilet PM, Kunreuther E, Contreras M, Cibas ES, Orcutt J, Moore FD, Jr, Larsen PR, Marqusee E et al. 2006 Prevalence and distribution of carcinoma in patients with solitary and multiple thyroid nodules on sonography. Journal of Clinical Endocrinology and Metabolism 91 3411-3417. (doi:10.1210/jc.2006-0690)

Fugazzola L, Puxeddu E, Avenia N, Romei C, Cirello V, Cavaliere A, Faviana P, Mannavola D, Moretti S, Rossi S et al. 2006 Correlation between B-RAFV600E mutation and clinico-pathologic parameters in papillary thyroid carcinoma: data from a multicentric Italian study and review of the literature. Endocrine-Related Cancer 13 455-464. (doi:10.1677/erc.1.01086)

Minuto MN, Miccoli M, Viola D, Ugolini C, Giannini R, Torregrossa L, Antonangeli L, Aghini-Lombardi F, Elisei R, Basolo F et al. 2013 Incidental versus clinically evident thyroid cancer: a 5-year follow-up study. Head \& Neck 35 408-412. (doi:10.1002/hed.22974)

Negro R, Piana S, Ferrari M, Ragazzi M, Gardini G, Asioli S, Pacchioni D, Riganti F \& Valcavi R 2013 Assessing the risk of false negative fineneedle aspiration cytology and of incidental cancer in nodular goiter. Endocrine Practice 21 1-24. (doi:10.4158/EP12271.OR)

Ugolini C, Giannini R, Lupi C, Salvatore G, Miccoli P, Proietti A, Elisei R, Santoro M \& Basolo F 2007 Presence of BRAF V600E in very early stages of papillary thyroid carcinoma. Thyroid 17 381-388. (doi:10.1089/thy. 2006.0305)

Xing M 2007 BRAF mutation in papillary thyroid cancer: pathogenic role, molecular bases, and clinical implications. Endocrine Reviews 28 742-762. (doi:10.1210/er.2007-0007)

Received in final form 13 June 2013

Accepted 18 June 2013

Made available online as an Accepted Preprint

18 June 2013
(C) 2013 Society for Endocrinology Printed in Great Britain 